\title{
The Future: Obstacles and Opportunities
}

\author{
Udo Kannengiesser
}

\section{Abstract}

This chapter discusses the possible future of using S-BPM in production industry, including prospective obstacles and potential opportunities. It commences by proposing a framework representing the fundamental values of S-BPM relevant for its contribution to production enterprises: agility. These values are derived from the agile approach to software development. It is shown how S-BPM supports them in several ways; specifically

1. Individuals and interactions are supported by the notational simplicity in S-BPM

2. Working software is supported by the ability of S-BPM to seamlessly integrate processes along life cycles and value chains

3. Customer collaboration is supported by the widely shared semantics of S-BPM modelling constructs

4. Responding to change is supported by the ability to encapsulate process functionalities by means of subjects in S-BPM

The principal obstacles are identified for the use of S-BPM in industrial practice, in a way to achieve the four agile values. They include a widespread perception of process modelling as a routine task (not a creative activity), security concerns for core production processes, organizational cultures where there is a strong sense of hierarchy and silo mentality, and a desire for global control flow. Based on the size of each obstacle and the degree to which S-BPM is already prepared to address them, the beginnings of a roadmap towards industrial fitness are then developed. For this purpose, the metaphor of a

U. Kannengiesser $(\bowtie)$

Metasonic GmbH, Pfaffenhofen, Germany

e-mail: udo.kannengiesser@gmail.com 
"compass" is introduced to give orientation to future S-BPM research within a four-dimensional space of opportunities. A specific S-BPM project in the food industry, as part of the SO-PC-Pro project, is presented to show common drivers and challenges of S-BPM implementations for production processes within this four-dimensional space. Finally, the compass is used for identifying further domains that share similar issues likely to be solved using an agile approach supported by S-BPM. The architecture-engineering-construction (AEC) domain is presented as an example of such a domain.

\subsection{The Fundamental Values of S-BPM in Production}

In order to predict the future potential for S-BPM in the production industry, it is useful to abstract from the specifics of the individual case studies presented in this book. This chapter outlines a framework that proposes four dimensions that characterize the essence of using S-BPM in the production industry. This framework will provide a basis for:

1. Identifying and classifying obstacles for adopting S-BPM in the production industry

2. Directing research activities and practical applications to seize opportunities in production and other, similar domains

How can we derive such a framework? In the previous chapters, S-BPM has been discussed in the context of two overarching goals in production companies: On the one hand, traditional production management needs to be enriched with humanistic aspects, such as worker empowerment and autonomy. On the other hand, production processes need to be automated using decentralized, highly flexible technologies for increased customer satisfaction. These two goals are consistent with an existing notion that has become popular in several industry domains, and is termed agility.

Common views of "agility" as such have not much in common with humanistic values. Superficially, this notion is often seen as synonymous to flexibility: the ability of a system to respond to change (Saleh et al. 2003). However, most of the approaches to achieving agility in socio-technical domains such as manufacturing, project management and software development are based on autonomous, local decision-making by individuals (i.e. people). Rather than having to adhere to central control mechanisms, people in agile systems are encouraged to utilize their own knowledge and creativity to respond to the situation at hand, and use communication and collaboration to align their individual decisions and actions with others.

This concept is closely associated with lean production systems, where individual production units (people or machines) communicate with one another via kanbans to streamline the overall flow of work. The connection between lean management and humanistic values has been made most explicit by the notion of kaizen. Kaizen implies a cooperative management style. Work is typically aligned 
among cross-functional, interdisciplinary work groups that collectively decide upon clear work goals and intensively exchange information for work alignment. Consensus among work groups instead of individual top-down management decisions represents a primary goal of Kaizen. Kaizen aims at employees who are highly qualified and who pro-actively and self-directedly contribute to workplace innovation and continuous improvement.

Given the dual nature of agility as a catalyst for producing not only humanistic but also economic value in socio-technical systems, it can potentially provide the basis of a generic framework for S-BPM in the production industry. One of the most known frameworks for agility has been proposed in the domain of software engineering: the "manifesto for agile software development" (Fowler and Highsmith 2001). It includes the formulation of four basic values that are shared across various techniques for agile development:

1. Individuals and interactions are valued more than rigid procedures and tools

2. Working systems are valued more than comprehensive documentation

3. Customer collaboration is valued more than contract negotiation

4. Responding to change is valued more than following a plan

The remainder of this section elaborates the four agile values including the support provided for each of them by S-BPM. This will provide a basis for identifying obstacles and opportunities for future applications of S-BPM in the production industry.

\subsubsection{Individuals and Interactions: Support Through Notational Simplicity}

Individual people and their interactions are considered as a critical success factor in the development of new systems, including production processes (Yauch 2007; Alves et al. 2012; Brauner and Ziefle 2015). It is the ability of individuals to reflect-in-action (Schön and Wiggins 1992), often through informal communication and self-organized teamwork that can lead to the discovery of new requirements and conceptual solutions, thus leading the design process in new directions (Gero and Kannengiesser 2014). This ability is needed mostly in development projects with high degrees of novelty and dynamics. In contrast, conforming to predefined, rigid processes can stifle the creativity needed for generating successful design outcomes.

IT tools may also turn out to be straightjackets for people, by requiring overly formal representations or by providing functionalities that are too complex and often unnecessary. In addition, most IT tools do not well support interactive, collaborative modes of working. They typically use conventional desktop screens with mouse and keyboard, granting full access to the tool only for a single person at the same time. Some tools provide more sophisticated collaboration features ranging from shared model repositories to virtual reality; however, they are still seen as inferior to collocated, physical human interaction with its rich set of gestures, facial expressions, etc. 
If individual creativity and informal interactions are to be supported, there needs to be a way to model production processes in a quick and easy way, without having to conform to many formal modelling conventions or to struggle with complex tool functionalities. The resulting models may be rough sketches "on the back of an envelope" that may even be incomplete or ambiguous. Their main aim is to provide a basis for discussion, reflection and reinterpretation, often among team members in spontaneous, informal meetings. For example, mechanical engineers rarely commence modelling a new product directly using a CAD tool; instead, they commonly produce hand-drawn sketches on paper, whiteboard or other physical surfaces, in order to reflect on their ideas individually or sharing with others. The importance of sketching activities in the conceptual design stages has been pointed out in a number of domains (Schütze et al. 2003; Petre 2009; Eckert et al. 2012).

A minimal requirement for supporting quick and easy sketching of production processes is the availability of graphical modelling languages, based on their ability to facilitate human understanding (Gerber et al. 2014). For example, the IEC 61131-3 standard contains three graphical languages for structuring and programming PLC code specifying low-level process control. A variety of BPM approaches provide graphical notations for modelling on the business process level. However, many of these languages are highly complex with very intricate syntax, requiring specialized modelling tools. On-the-fly sketching of processes, either manually or by low-tech physical tools, would be quite difficult using these languages.

S-BPM provides the notational simplicity required for quick and easy sketching. Since it has only five modelling constructs, their visual syntax can be defined in a way that allows easily distinguishing them from each other. Perceptual discriminability is one of the key factors for the cognitive effectiveness of visual notations (Moody 2009). For example, the syntactic elements used in the sample models of the principal reference book on S-BPM (Fleischmann et al. 2012) are perceptually discriminable based on simple shapes. The S-BPM tool Metasonic Suite uses colour as an additional visual variable to further enhance discriminability between the syntactic elements. Producing five basic shapes or colours for modelling can be done using any common sketching tool, such as pen and paper, whiteboards, flipcharts and post-it notes. In addition, many of these tools support interactive ways of working within teams of modellers, which enhance modelling outcomes by stimulating discussions and learning (Rosemann 2006).

\subsubsection{Working Systems: Support Through Seamless Integration}

One of the major characteristics of agile projects is the continuous, frequent development of working systems in small increments. The notion of a "working system" here goes beyond being just "bug-free"- the system needs to "work" in terms of being useful for an individual solving a specific task (Bider 2015). One of the reasons for this emphasis on working systems is to shorten feedback loops, thus reducing the risk of developing a system that does not properly address the needs 
and expectations of its users. Deploying and using working systems in their intended environment is seen as the most effective basis for such an assessment. It allows users actually "seeing" the impact that the new software has on their task processing and problem solving. This can also be considered the foundation of the well-known "Plan-Do-Check-Act" cycle for continuous improvement in lean production (Dennis 2016). Documentation, such as requirement specifications, system models and test reports, can supplement but not replace the first-hand experience of interacting with the running system.

What is required to support the creation of working production systems once a production process has been modelled? Concerning the creation of control software, model-driven development (MDD) (Mellor et al. 2003) is the notion that addresses part of this issue. Here, graphical process models are transformed automatically into a representation that can be directly executed, e.g. by a workflow engine or a PLC. Another notion supporting the creation of running production systems is enterprise integration. A single process execution engine rarely operates in isolation: In most real-world applications there is an existing IT environment that needs to interact with the execution of a process. Examples include ERP systems, databases and other workflow engines, which may exchange various kinds of data with the process. What is needed, is a mechanism for data integration with external systems, preferably using existing interoperability standards depending on the domain.

S-BPM supports the creation of working systems in production companies by seamless integration in the sense of both model-driven development and enterprise integration. Specifically, S-BPM models can be transformed into two types of executable representations: Abstract State Machines (ASM) (Börger and Stärk 2003) for business process execution by subject-oriented workflow engines, and IEC 61131-3 Sequential Function Charts (SFC) (Müller 2012) for real-time execution by PLCs. S-BPM also provides a number of ways to exchange data with external systems, such as ERP (Dirndorfer 2015), MES (Kannengiesser et al. 2016) and PLCs (Kannengiesser et al. 2015).

\subsubsection{Customer Collaboration: Support Through Widely Shared Semantics}

Closely involving customers in the development phase aims at producing a clear, common understanding of the requirements. The notion of "customer" should be understood in a broad sense, including the concrete adopters of the system or service to be designed. Adopters may be end users or service consumers within value networks. Customer collaboration then implies that adopters are engaged to participate in the specification and design of the system throughout the project. They are viewed not merely as the final recipients of the system but rather as development partners (van Aken 2007). The kaizen approach in lean manufacturing makes extensive use of this concept, by encouraging workers to participate regularly in the improvement of processes and their organization (Berger 1997). 
The concept of customer collaboration aims at maintaining a shared agreement about the requirements and system features as the development progresses, encourages stakeholder participation during system design and testing, and increases acceptance of the system during usage. Contract negotiation is the opposite approach, because it limits stakeholder involvement to a separate, upstream phase of requirements specification. A requirements document is produced that is treated as a "contract", implying an ultimate character and discouraging any changes after it has been specified. Such a rigid, top-down approach is rarely in line with the dynamics of reality. In addition, its nearly exclusive reliance upon written specification documents can easily lead to misunderstanding.

For all stakeholders, including production managers, engineers and workers, in order to participate effectively in process development, they need a common language. The semantics of such a language needs to be understandable independently of the stakeholders' levels of expertise and domain specialization. The simple semantics of S-BPM drawn from human communication and organizational theory can provide a good foundation for such a language, despite a few difficulties currently remaining in its practical use (see Chap. 7 "Learnings").

For subjects executed by human actors, such a semantics appears intuitive as it matches the individual's perception of organizational reality: One can either do something (represented as function states), send messages (represented as send states), or receive messages (represented as receive states). Even when subjects are executed by software or machines, the cognitive effort needed to conceptualize their interactions in terms of communicative actions can be assumed to be relatively lowusing anthropomorphic metaphors is a common human strategy for understanding and predicting an agent's behaviour (Dennett 1987; Wooldridge and Jennings 1995).

\subsubsection{Responding to Change: Support Through Encapsulation}

Rather than following a fixed development plan, it is often more effective to accept that changes to the plan will occur. Most instances of designing are iterative and frequently involve reformulating requirements and subsequent changes in the trajectory of designing (Gero and Kannengiesser 2014). There are numerous types of design iterations (Wynn et al. 2007). They are driven mostly by the introduction of new business requirements or technical constraints, the discovery of unforeseen design problems, and the emergence of new design opportunities (Schön and Wiggins 1992).

A common way to prepare for change in system design is the concept of modularity. The fundamental idea is to reduce dependencies between system components by structuring them according to distinct functional modules. The result is a loosely coupled system architecture that allows substituting individual modules with no or only limited impact on other modules in the system (Ulrich 1995; Baldwin and Clark 2000). The same idea has been applied to services and processes including production processes (Bask et al. 2010). While the ideal degree 
of modularity varies according to the specific context of the system (Schilling 2000; Schilling and Steensma 2001), the underlying principle for inducing any form of modularity in a system is encapsulation. Encapsulation allows separating different functionalities among each other as well as from structural components (i.e. physical mechanisms, human or computational agents that provide these functionalities) (Gero and Kannengiesser 2003; Kampert and Epple 2014).

The notion of a subject in S-BPM fits with the idea of encapsulation. Subjects encapsulate different functionalities by exposing only their inputs and outputs (i.e. the messages they receive and send) while hiding their internal behaviour. This allows modifying this behaviour without affecting the rest of the process as long as the inputs and outputs remain the same. Encapsulation in S-BPM is also applied regarding the distinction between functionality and structural components, because subjects are clearly separated from the agents executing them (Fleischmann et al. 2013). As a result, agents can be substituted without changing the process model; only the mapping between subjects and agents needs to be modified.

\subsection{Obstacles}

The concept of agility is well known in the production industry. Together with its sibling, lean production, it has also been very popular in various other domains. Today many companies claim that they are agile or provide agile solutions. However, at closer inspection their understanding of agility is often quite shallow, limited to providing some form of flexibility in their products and services. As our project could reveal, truly agile values, such as the ones described in this chapter, are rarely lived in the domain of process management, especially in traditional manufacturing firms. What are the reasons for this lack of adoption? Answering this question will directly shed light onto potential obstacles for using S-BPM in production.

In this section we will use the four agile values articulated in this chapter as a framework for identifying and classifying fundamental obstacles for using S-BPM in production.

\subsubsection{Process Modelling as Routine Task not Ideation}

The predominant purpose of process modelling today is documentation (Kocbek et al. 2015) — which can be presumed to be a routine task that involves rather little creativity. This fits with some of the terms used in literature and practice: Processes are often referred to as being "modelled" or "mapped" rather than "designed". A Google Scholar search in August 2016 returns 27,540 English articles for the term "business process modeling" and only 6,700 for "business process design". Based on the perception of process modelling (or design) as a routine task, highly structured, systematic approaches are commonly preferred over less structured ones 
such as design thinking. This fits with observations that design thinking techniques have been rarely adopted in enterprise IT despite their popularity in product design (Gartner 2015). This is especially the case for IT in the production industry, where company cultures, organizational structures, and work procedures are oriented rather to traditional engineering approaches.

Understanding process modelling as a documentation or requirements definition method has led to the development of comprehensive process notations such as BPMN that aim to support a high degree of expressiveness. BPMN, today the most commonly used approach in BPM (Harmon 2016), requires a complex syntax that is not easy to be learned and applied (Recker 2010). Since there is a correlation between process modelling competence and the creativity of process modelling outcomes (Figl and Weber 2012), it can be assumed that the lack of adequate BPMN expertise of many practitioners affects their creativity in process modelling negatively. In addition, the complex notation of BPMN requires computational tool support for modelling, which may explain the apparent importance of process modelling tools in BPM surveys (Harmon 2016). It is certainly possible to sketch process models using only a core or subset of the most important modelling elements in BPMN (Recker 2010; Grosskopf et al. 2010). Yet, the resulting models would then have to be interpreted by modelling specialists to manually transform them into more complex diagrams using a more complete subset of the BPMN specification, and using the BPMN modelling tools that many companies already invested in. This approach is error-prone as it bears the risk of misinterpretation.

The widespread view of process modelling as a routine task, reinforced by the current dominance of the BPMN approach that has been declared a standard for BPM, clearly favours procedures and tools over individuals and interactions. This is an obvious obstacle for the adoption of S-BPM, particularly in rather conservative domains such as the production industry.

\subsubsection{Don't Mess with My Core Process}

Although the seamless integration of production and business processes is the declared goal of numerous research initiatives and standard committees, companies often remain wary regarding this topic. The reason for that is the belief integrating processes also means exposing them, thus making them potentially vulnerable to privacy and security threats. Traditionally, many manufacturing organizations have sought to protect their production processes by disconnecting them from the outside world. This shift especially concerns their IT systems used for lower-level automation control, as these systems are considered to be vital for manufacturing operations. Any malfunctioning of these systems, e.g. as a result of denial-of-service attacks, will directly incur loss of productivity and loss of revenue (Sadeghi et al. 2015). A maximum level of security is therefore preferred, often by physically isolating security-critical systems and "core processes" from the rest of the enterprise. This strategy is known as the "air gap" principle (Lass and Kotarski 
2014). Another strategy for keeping core production processes secure has been the use of proprietary systems without standard interfaces, hoping the effort involved in finding and exploiting vulnerabilities of a system will be too high for successful attacks.

To realize the benefits of Industry 4.0 business models, there need to be seamless integration mechanisms that address concerns of privacy and security. Some of these mechanisms are already provided by standard communication protocols such as OPC UA (Hoppe 2014). Yet, they need to be complemented by protective measures on the business process level.

Today S-BPM does not offer solutions to these issues. The degree to which core processes are exposed to external systems is often limited to coarse-grained documentation, whereby simple flowcharts are generally preferred over executable notations such as S-BPM.

\subsubsection{Hierarchies and Silos}

A wide variety of stakeholders may need to interact at different stages of the life cycle of production systems and production processes. They include mechanical engineers, electrical engineers, software specialists, production managers, product designers, shopfloor workers and sometimes clients. Discussing different process designs in terms and models that can be understood by all stakeholders independently of their education and discipline-namely, by using a common language such as S-BPM-would certainly be advantageous. However, the main obstacle here is that the local work culture in many companies does not encourage collaborative ways of working. For example, it can often be observed that managers take process design decisions without including shopfloor workers in the decision-making process.

In addition, mechanical engineers often devise production systems and processes without consulting with the software specialists implementing associated control systems (Alvarez Cabrera et al. 2010). These two examples refer to cultural issues that can be referred to by the notions of hierarchies and silos, respectively. Companies with a strong sense of hierarchy rarely use management approaches that feature worker participation and empowerment. Silo mentality represents a similar obstacle leading to poor collaboration across disciplines, functional departments and business units.

The ground for S-BPM in production seems to be most fertile where companies already have established lean and open organizational cultures. This finding is confirmed by numerous industry experts viewing Lean Management as a precondition for the successful implementation of Industry 4.0 and smart factories. Regarding process modelling aspects of Lean Management, Kannengiesser (2014) has already shown the consistency of S-BPM with the value stream design (VSD) approach. However, the actual challenge remains for company organizations, namely to keep pace and align with these technical advances and foster a participative, collaborative work culture. 
Where work cultures are characterized by hierarchies or silos, a strong tendency exists to minimize and/formalize collaboration across interfaces between functional units. Such a tendency favours "contract negotiation" and discourages open collaboration, establishing a primary obstacle for adopting S-BPM.

\subsubsection{The Desire for Global Control Flow}

A common human strategy for understanding and analyzing complex systems is to construct simplified models representing them. These models concentrate on the most typical case, ignoring many variations and exceptions that may occur. Complex processes are mapped into linear sequences of abstract activities, even though in reality stakeholders may behave non-linearly and in unpredictable ways. The fact that these models often remain at quite a low resolution and at best provide a snapshot of reality has commonly been accepted as an instance of the Pareto principle: $80 \%$ of the benefits of process modelling stem from $20 \%$ of the process modelling effort. However, as business and production become more volatile and heterogeneous (Sinur et al. 2013), this principle does no longer apply-at least not by focussing on just $20 \%$ of all process variants. Unfortunately, this issue has remained unnoticed by many process managers. For them, process models need to be linear and mostly sequential, preferably from "end to end".

S-BPM does not provide or support such a linear perspective. It conceptualizes processes as interacting subjects that encapsulate behaviour, and thus hide parts of the process. Instead of following a centralized (and thus linear) control flow, the interactions between subjects can occur indeterministically at execution time. The interplay between subjects is not represented as a linear flow of activities but as an unordered network of messages in a Subject Interaction Diagram. S-BPM models are thus oriented towards local autonomy and behaviour of the agents that execute the subjects, and towards the ability to modify individual behaviours without necessarily affecting the whole process system. There is not much work on combining this bottom-up view with a top-down view describing a process from a global system perspective. In such a perspective, the focus is on the desired sequence of tasks to be performed, in order to achieve the system's goals. It may partially explain the ongoing popularity of simple flowcharts and control flow-oriented approaches such as BPMN. Representations capturing a process in terms of an "end-to-end" sequence of tasks have not been in the focus of research in S-BPM till date.

What is missing in S-BPM models to provide a global process view? According to some BPM practitioners, S-BPM lacks constructs that explicitly show the logical sequence of (main) tasks (as, e.g. represented by sequence flow in BPMN), the exact location(s) of the end of the process (as represented by "end events" in BPMN), and the location of key decision points within the process (as represented by gateways in BPMN). These would be models where the order of activation of subject instances during process execution can be defined according to an assumed "happy" (or any other, specifiable) path. 
The desire of "following a plan" as suggested by centrally controlled, linear process models is a clear obstacle for applying S-BPM. At present, there are no subject-oriented constructs that can cater to the desire for global control-flow modelling.

\subsection{Opportunities}

We have elaborated the values and generic benefits of S-BPM as well as its major obstacles in the production industry. Given this situation, what are the opportunities that arise, in terms of areas of research and industry where S-BPM can realistically create an impact? In this section, we try to provide an answer by first developing the beginnings of a roadmap for S-BPM in production, before presenting a case study overcoming some of the obstacles, and examining other fields of application beyond the classical view of "production".

\subsubsection{Towards a Roadmap for Using S-BPM in Production}

Having identified the various obstacles for adopting S-BPM in the production industry, it is possible to develop a roadmap that may help navigating around these obstacles. In this section, we will outline an initial basis for such a roadmap.

The four agile values are not completely independent of each other. However, for the purposes of building a roadmap, we can treat them as four orthogonal dimensions to provide future research with a frame of reference. Driving research along one dimension means to develop extensions of S-BPM (methodologically or computationally) and/or evaluate these using industrial case studies. Each of these research efforts will face different obstacles as outlined in the previous Sect. 8.2. The size of each obstacle and the degree to which S-BPM research is already prepared to tackle them determines the speed with which research can demonstrate the overall benefits of S-BPM in production. A roadmap may use this information to propose a research agenda that aims at reaching out for the "low-hanging" fruit first and addressing the more challenging issue later.

Figure 8.1 provides an overview of the four research dimensions using the concept of a compass. Contrary to the normal use of a compass allowing two-dimensional navigation, we use this concept to allow navigating in four dimensions. So, in our four-dimensional world, "navigating" towards notational simplicity ("North") does not mean moving away from seamless integration ("South"). Our "compass" is thus an abstract metaphor for navigation to help visualizing opportunities for future research, despite the potential misunderstanding pointed out here.

The circular arrow in Fig. 8.1 represents the sequence in which the four dimensions ought to be addressed for boosting the adoption of S-BPM in 
Fig. 8.1 A "compass" for S-BPM in production industry

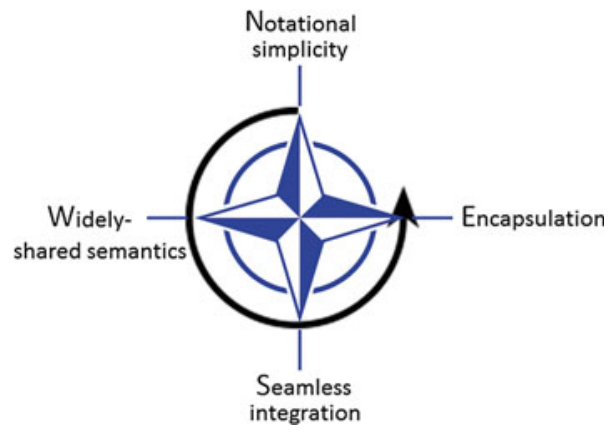

production industry. We chose the indicated sequence based on the amount of effort we perceive will be required for tackling the associated obstacles.

The least amount of research effort is likely to be needed for removing or navigating around the obstacle of "process modelling as routine task not ideation", thus driving research towards the notational simplicity dimension ("North" in the compass in Fig. 8.1). Even in cases where process modelling is perceived a routine rather than a creative task, the benefits of S-BPM in terms of ease of use and stakeholder engagement are immediately obvious, as indicated by a growing number of field studies in various industries (Fleischmann et al. 2015). More of these studies are needed showing these benefits in the production domain.

Work in the SO-PC-Pro project has already started to deliver such case studies, having the potential to serve as reference cases for further applications. Especially in the more technical process domains in production where the BPMN standard had only limited influence so far, there is a good chance that S-BPM may be welcomed more than in traditional business process management domains. This effect may be leveraged by future initiatives aiming to transform S-BPM into a formal standard endorsed by an international standards committee. Another strategy could be to borrow a limited set of graphical elements from the BPMN notation but constrain their use to match the modelling semantics and conventions of S-BPM (Turetken and Demirors 2013; Fichtenbauer and Fleischmann 2016).

The obstacle of "hierarchies and silos" is a slightly trickier one to address, as it is a more general problem. Fortunately, an increasing number of companies are adopting open work cultures. They are the ones S-BPM practitioners can directly target rather than facing an uphill battle with traditional company cultures. The S-BPM approach provides them with a tool that can overcome silos and hierarchies based on its widely shared semantics ("West" in the compass). What still needs to be addressed in more detail, however, is a governance framework answering questions such as: When should a particular stakeholder become involved? To what extent? Should the modelling activity be performed top-down or bottom-up? Such a framework could be used to develop guidelines to help process managers feel more at ease with the S-BPM modelling approach as today it does not answer these practical questions. 
The next obstacle to be addressed is the one we call "don't mess with my core process", impeding progress towards seamless integration ("South" in the compass). It is based on the fundamental security, safety and privacy concerns that persist in the production industry and thus keep raising reservations against technologies enabling seamless integration. S-BPM will not be able to dissolve these concerns in the short term, as significant amounts of research as well as tool developments are necessary. S-BPM does provide a sound conceptual basis for this research, as it contains a number of concepts that can be used for enhanced privacy and security control in seamlessly integrated production processes. Particularly, the concept of encapsulation in S-BPM may be used as a basis for effective protection of processes and data from external threats. Such an approach requires the implementation of S-BPM extensions as proposed by Dirndorfer et al. (2012). In addition, sophisticated mechanisms for access control of subjects (Lawall et al. 2015) need to be realized.

Probably the most challenging obstacle to address is "the desire for global control flow" that hampers adoption of the encapsulation idea ("East" in the compass). A number of extensions of S-BPM seem to be necessary. Possible research avenues include modelling incomplete or more coarse-grained subject behaviours, similar to the notion of "normalized" behaviour proposed by Fleischmann et al. (2012). Future work may also require a way to turn communication-based subject relations into more abstract control-flow relations. This enrichment could reduce the "communication clutter" caused by the typically large number of messages in S-BPM models that often reduces readability, and could provide a more condensed visualization of the main functionalities in the process and their (expected or desired) sequence. Another way could be to introduce the notion of a process view: Modellers can define and switch between different views of the same process, depending on the specific purpose (Browning 2009). For example, one may define a view of a Subject Interaction Diagram where only those messages are shown that are associated with the value stream (Kannengiesser 2014); other messages solely aiming at coordinating different subjects would then be omitted. This reduces the number of messages in a chosen view without having to resort to control-flow diagrams. Another view of a Subject Interaction Diagram may use the design structure matrix (DSM) representation of processes (Kannengiesser 2015).

Each of the four dimensions may be elaborated in future work, e.g. by adding specific milestones. This could guide research activities in the sense of a detailed roadmap and would allow measuring their progress.

\subsubsection{Practical Application: A Case Study in the Food Industry}

The compass introduced in Sect. 8.3 represents the beginnings of a roadmap for research in S-BPM in production, suggesting a sequence in which the four principal issues can be addressed. We can adapt the meaning of this compass to identify the 
drivers and challenges related to using S-BPM in specific process implementation projects in the production industry. In this section, we show how these challenges were dealt with in a case study in the food industry.

The case study has been implemented for a large manufacturer of baked products based in Mexico. In this chapter we will refer to this manufacturer as Company $\mathrm{C}$. Through participating in SO-PC-Pro, the management of Company $\mathrm{C}$ wanted to perform a process improvement project in one of its sales outlets as a pilot for rolling out the solution in other outlets throughout Mexico. The main focus was increasing the profitability of the branch. For this purpose, two value drivers were identified:

1. Loss of revenue because of product returns (i.e. products that need to be discarded as they are no longer fresh (shelf life exceeded))

2. Loss of revenue because of lack of products (i.e. missed sales opportunities)

Targeting these value drivers were the main goals of the case study. As such, the case study can be seen as a Lean improvement project, as it follows the classical principle of the Lean methodology: to smoothen process flow by eliminating various kinds of "waste"; here, the wastes of overproduction (generating unnecessary stockwhich in the present case study needs to be discarded after reaching the end of their shelf life) and underproduction (causing consumers to wait for production-which in the case study manifests itself as empty shelves and disappointed customers).

S-BPM was used for developing an improved production and delivery process for baked and frozen goods in the selected sales outlet. This process implements a pull system - a well-known Lean design principle - in addition to the existing push system. This means that production is controlled not only by an upfront schedule or production plan ("pushing" the process) but also by variations in product demand ("pulling" the process). The process was partially automated and integrated in the sales branch. Previously this process was executed only manually, without being enforced or supported by a process execution system.

The four challenges or dimensions identified for S-BPM had various effects on the project.

Notational simplicity: The relative ease of using S-BPM enabled interdisciplinary team of ten people to produce and validate a complete, executable model of the to-be production process within a combined training and modelling workshop that lasted five days. The modelling activity was initially performed using post-it notes, cards and flipcharts, as shown in Fig. 8.2. Most workshop participants were included in these initial stages of modelling, confirming the playful, engaging character of S-BPM modelling. All models resulting from these initial elicitation sessions were later transformed into computational models using the S-BPM tool Metasonic Suite.

Apart from two S-BPM experts in the project team, none of the other team members had modelled with S-BPM prior to the project. However, they were all familiar with flowcharts, and two of them had even worked with BPMN in previous 


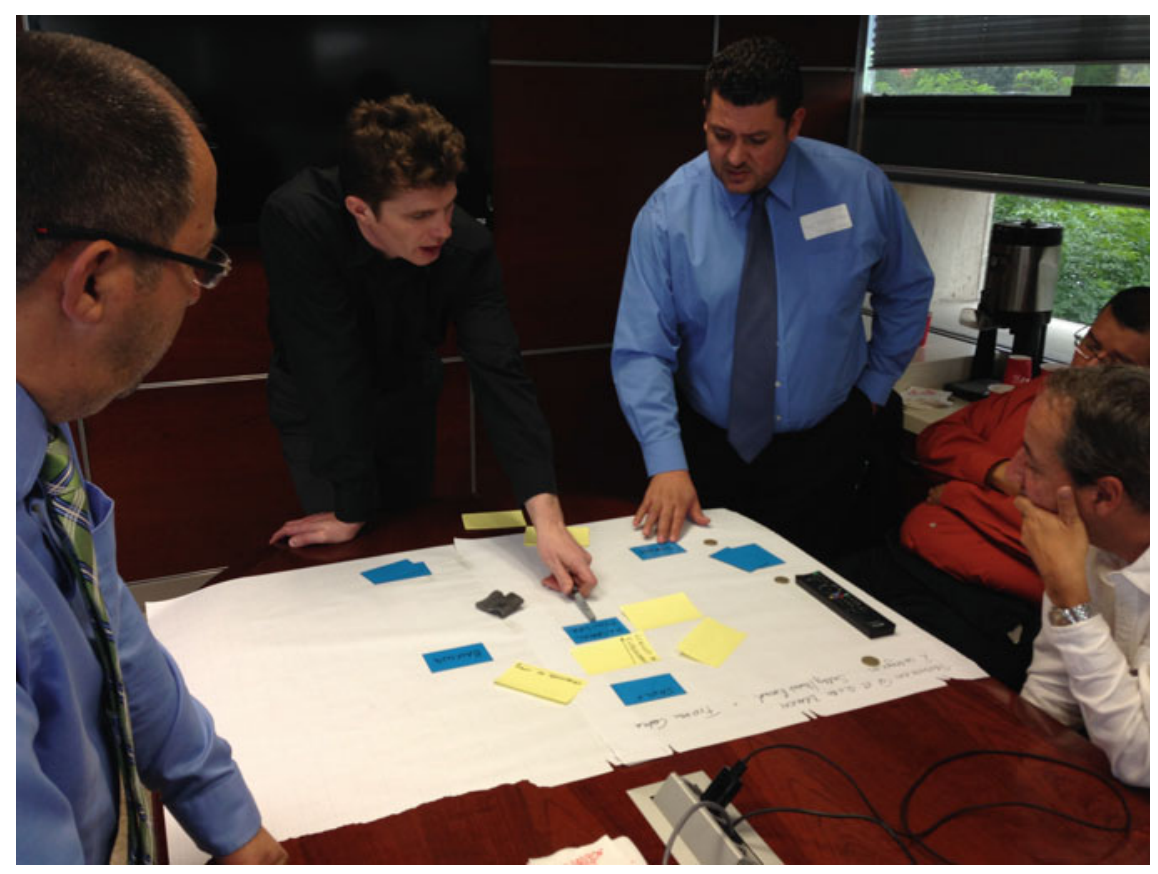

Fig. 8.2 Initial S-BPM modelling session performed using physical tools

projects. Despite this bias towards the control-flow paradigm, everyone in the team was fairly quickly able to get used to the subject-oriented way of thinking.

Widely shared semantics: Eliciting the production process from the workers in the case study followed a traditional approach using interviews and observations by consultants. Workers were not directly involved in creating, verifying or discussing the process model. It was only through pilot testing of the implemented process with its associated execution support systems that workers had the occasion to experience and comment on the process. The lack of worker involvement during the modelling stage was partially due to the technical character of the project, which mainly aimed at automating tasks rather than establishing a completely novel way of working. The creation of the process model was guided by two S-BPM experts, overcoming the lack of formal frameworks governing the S-BPM modelling activities. They also managed to train the S-BPM novices in the team to conceptualize processes in terms of the five simple constructs: subject, message, function state, receive state and send state.

Seamless integration: For the purposes of the project, processes were not required to be integrated vertically. The only possible integration that was discussed was horizontal: Should the new production process include the upstream processes in the plant providing the raw materials and producing unfinished products? A decision was made to leave these (core) processes out of the scope of this project, 




Fig. 8.3 Flowchart providing a simplified, global view of the production process

due to the nature of the project as a research endeavour. Security and similar concerns would have been likely to play a role if this decision had been the other way.

Encapsulation: The outcomes of the initial phase of process elicitation-before the S-BPM modelling approach was applied in the project-were captured using a simple flowchart representation as shown in Fig. 8.3. This flowchart includes icons and basic shapes with fairly open semantics. It was used to represent the production process at a high level of abstraction, without enforcing any formal modelling conventions. This flowchart was helpful in generating a common understanding about the process among project team members. However, when using the S-BPM approach for modelling the same process, the flowchart was no longer used. The change in thinking from control flow towards subject-orientation required some cognitive effort from some team members, which was facilitated by the two S-BPM experts in the team.

The case study demonstrated the strengths and challenges of S-BPM according to the four dimensions. While the implementation and evaluation of the case study is still ongoing, the experiences gained during this study indicate that S-BPM is a promising approach for process improvement projects in the production industry although open research issues remain.

\subsubsection{Other Fields of Application: Architecture-Engineering-Construction (AEC) as an Example}

The high generality of the compass in Sect. 8.3.1 allows its application in domains not directly related to production but sharing similar concerns including the need for agility. These may be domains situated along the value chain or the lifecycle of produced goods and services (ARC Advisory Group 2001). For example, domains such as product data management (PDM), product lifecycle management (PLM), supply chain management (SCM) and customer relationship management (CRM) may require applications supported by an agile approach to process management. Other domains may be located even further from manufacturing. One of them is the architecture-engineering-construction (AEC) industry. This Section will present AEC projects as an example for the potential use of S-BPM in domains beyond production. 
One of the principal concerns in the AEC domain is the planning and execution of construction projects for the built environment. Over the past two decades, there has been an increasing focus on the digitalization of building data to speed up the execution of construction projects. The research concerned with digitalization in this domain is commonly known as Building Information Modelling (BIM). A lot of research and development activities in BIM have been devoted to creating a standard model for building information, called Industry Foundation Classes (IFCs). This standard comprises hundreds of building concepts for all building lifecycle phases (e.g. design, construction, management and demolition). Its aim is to support interoperability across hundreds of software applications and industry domains. Subsets of IFCs, called Model View Definitions (MVDs), can be specified to allow swift access to relevant parts of the data standard.

To facilitate the practical use of IFCs, the approach of Information Delivery Manuals (IDMs) has been developed and adopted as an international standard (ISO 29481-1:2010). IDMs specify how data can be exchanged among different project stakeholders by means of a process model and the types of information to be exchanged. Specifications contained in an IDM are then mapped to relevant MVDs. Specifically, the following artefacts are contained in an IDM:

- Process Map (PM): It is used for defining the industry process to be supported. It should contain a set of activities, roles and the required data inputs and outputs.

- Exchange Requirements (ERs): They specify the information to be exchanged among the contractors.

- Functional Parts (FPs): They allow mapping IDMs to concepts in MVDs.

AEC projects are typically carried out in timeframes of several years (Eastman 2014). As one of the causes for long project durations one can identify the paper-based definition and exchange of IDM artefacts such as PMs, ERs and FPs. The manual work required for interpreting, maintaining and validating these paper documents is error-prone and time-consuming, especially when many parties are involved as typical for AEC projects. The method that best describes this way of working is the waterfall approach. In this approach, projects are structured in distinct phases that are separated by stage gates. The typical phases of an AEC project (with their respective timeframes) are shown in Fig. 8.4.

The waterfall approach allows iterations within a phase but discourages iterations across different phases: Once a document is produced at the end of a phase, it is regarded as a final agreement serving as a "contractual" basis for the subsequent

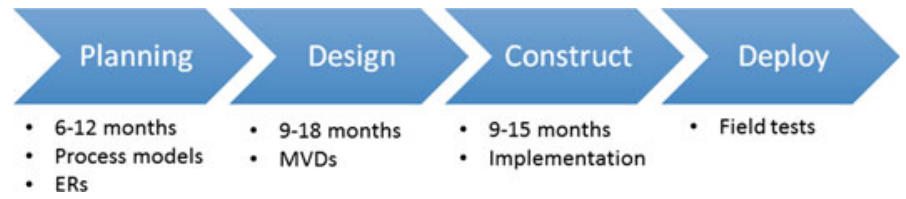

Fig. 8.4 Typical structure of an AEC project (based on Eastman 2014) 
phase. Later changes would require not only renegotiation among the parties involved, but also considerable effort to update and validate the consistency of IDM documents.

It is somewhat surprising that most AEC projects rely almost exclusively on paper-based documents, given that the principal motivation for BIM has been the digitalisation of models and model exchange. So far, the benefits of digital information exchange have been realized only for AEC process applications, but not yet for the development process that generates these applications.

One of the obstacles for digitizing data exchanges in AEC projects is the reliance on the traditional view of process modelling that favours extensive documentation over rapid design and testing. This is revealed by the IDM standard that recommends BPMN for defining process maps. However, some AEC researchers are increasingly interested in more agile AEC project approaches. Such research efforts can be categorized according to our four dimensions.

Notational simplicity: Lee et al. (2013) propose a method for IDM process modelling that restricts the use of BPMN for process modelling to a subset of only 22 notational elements. The authors have realized that "IDM development is challenging and error-prone due to an excessive and overwhelming number of BPMN shapes" (Lee et al. 2013, p. 649).

Widely shared semantics: Efforts to reduce the notational complexity of process models for AEC projects are also associated with the need to foster better collaboration between the various disciplines involved in these projects. Engineers, architects, constructors, facility managers, etc. should all be able to create, understand and give feedback on process models representing their roles in a project. This requires a common language, whose definition is the goal of current standardization initiatives such as building SMART (http://buildingsmart.org).

Seamless integration: The method proposed by Lee et al. (2013) tightens the connection between PMs, ERs, FPs and MVDs, to support an integrated, seamless development of process and data models. The use of standardized formats for MVDs, such as the mvdXML format, enhances the validation of IFC files against ERs defined in a given IDM and the corresponding MVD. The seamless integration supported by that work is limited to the initial stages of process development, unlike in production where seamless integration is mostly understood to cover the usage stage (i.e. the execution) of processes.

Encapsulation: Encapsulation in process models is not a well-known concept in IDM-related research. However, the strong interest in representing data flow (often represented in existing IDMs using message flow between separate BPMN pools) in IDMs may provide a fertile ground for adopting this idea. Subjects (i.e. encapsulations) and messages (interlinking subjects) can be seen as two sides of the same coin: Whenever there are several subjects in a process, there need to be messages to coordinate their behaviours.

S-BPM has recently been suggested as a means for increasing the agility of AEC projects (Kannengiesser and Roxin 2016), articulating the strengths of S-BPM with respect to the four dimensions. Yet, more work is needed to understand the potential implications of applying this new approach in the AEC domain. 


\subsection{Conclusion}

This chapter started by extracting the essence of using S-BPM in the production industry, namely as a vehicle for agility in the development of production processes. Borrowing from work on agile software development, four fundamental features of agility were proposed, and it was shown how S-BPM can support them. For each feature, a main obstacle was identified. It provided the basis for a roadmap of research in S-BPM in production, represented as a "compass" in a four-dimensional space of opportunities.

A case study in the food production industry was presented to illustrate the practical implications of using S-BPM in production. Challenges in the case study were classified and described according to the four dimensions of the compass. Finally, the potential usefulness of the framework beyond production was exemplified based on a description of typical shortcomings of architecture-engineeringconstruction (AEC) projects. The intention of this work was to outline the potential role of S-BPM in tomorrow's factories and give a possible pathway towards its adoption in the manufacturing and other domains. So far, the potential benefits of the subject-oriented approach have been pointed out for production industries mainly on a conceptual level. The case studies presented in this and other chapters are only beginning to demonstrate its practical value. This is one of the reasons, why S-BPM is still rather unknown in the world of production management. However, this situation is likely to change once a larger number of industrial applications using S-BPM are available that go beyond laboratory prototypes and research pilots, and prove its practical value. The conceptual framework established in this chapter can guide the development and evaluation of such applications.

\section{References}

Alvarez Cabrera, A. A., Foeken, M. J., Tekin, O. A., Woestenenk, K., Erden, M. S., De Schutter, B., et al. (2010). Towards automation of control software: A review of challenges in mechatronic design. Mechatronics, 20(8), 876-886.

Alves, A. C., Dinis-Carvalho, J., \& Sousa, R. M. (2012). Lean production as promoter of thinkers to achieve companies' agility. The Learning Organization, 19(3), 219-237.

ARC Advisory Group. (2001). Collaborative manufacturing management strategies. White Paper, October 2001. Retrieved August 19, 2016, from http://www.arcweb.com/arcreports2001/ Collaborative\%20Manufacturing\%20Management\%20Strategies.pdf.

Baldwin, C. Y., \& Clark, K. B. (2000). Design rules: Volume 1. The power of modularity. Cambridge: MIT Press.

Bask, A., Lipponen, M., Rajahonka, M., \& Tinnilä, M. (2010). The concept of modularity: Diffusion from manufacturing to service production. Journal of Manufacturing Technology Management, 21(3), 355-375.

Berger, A. (1997). Continuous improvement and kaizen: Standardization and organizational designs. Integrated Manufacturing Systems, 8(2), 110-117. 
Bider, I. (2015). Agile research in information systems field: Analysis from knowledge transformation perspective. In Proceedings of 8th IADIS International Conference on Information systems, Madeira, Portugal.

Börger, E., \& Stärk, R. (2003). Abstract state machines: A method for high-level system design and analysis. Berlin: Springer.

Brauner, P., \& Ziefle, M. (2015). Human factors in production systems: Motives, methods and beyond. In C. Brecher (Ed.), Advances in production technology (pp. 187-199). Lecture Notes in Production Engineering. Springer.

Browning, T. R. (2009). The many views of a process: Toward a process architecture framework for product development processes. Systems Engineering, 12(1), 69-90.

Dennett, D. C. (1987). The intentional stance. Cambridge: MIT Press.

Dennis, P. (2016). Lean production simplified: A plain-language guide to the world's most powerful production system (3rd ed.). Boca Raton: CRC Press.

Dirndorfer, M. (2015). ERP integration in S-BPM processes. In A. Fleischmann, W. Schmidt, \& C. Stary (Eds.), S-BPM in the wild: Practical value creation (pp. 257-268). Springer.

Dirndorfer, M., Handy, B., Schneeberger, J., \& Fischer, H. (2012). Subjective security and safetyS-BPM as a base for the description of security and safety objectives. In S. Oppl \& A. Fleischmann (Eds.), S-BPM ONE 2012, CCIS 284 (pp. 214-219). Berlin: Springer.

Eastman, C. M. (2014). Business process re-engineering for BIM: New directions in supporting workflow exchanges in IFC. In Building SMART alliance 2014 National Conference.

Eckert, C., Blackwell, A., Stacey, M., Earl, C., \& Church, L. (2012). Sketching across design domains: Roles and formalities. Artificial Intelligence for Engineering Design, Analysis and Manufacturing, 26(3), 245-266.

Fichtenbauer, C., \& Fleischmann, A. (2016). Three dimensions of process models regarding their execution. In $S$-BPM $O N E^{\prime} 16$. New York: ACM Press.

Figl, K., \& Weber, B. (2012). Individual creativity in designing business processes. In M. Bajec \& J. Eder (Eds.), CAiSE 2012 Workshops (Vol. 112, pp. 294-306). LNBIP. Berlin: Springer.

Fleischmann, A., Schmidt, W., Stary, C., Obermeier, S., \& Börger, E. (2012). Subject-oriented business process management. Berlin: Springer.

Fleischmann, A., Schmidt, W., \& Stary, C. (2015). S-BPM in the wild: Practical value creation. Berlin: Springer.

Fleischmann, A., Kannengiesser, U., Schmidt, W., \& Stary C. (2013). Subject-oriented modeling and execution of multi-agent business processes. In 2013 IEEE/WIC/ACM International Conferences on Web Intelligence (WI) and Intelligent Agent Technology (IAT), Atlanta, GA (pp. 138-145).

Fowler, M., \& Highsmith, J. (2001). The agile manifesto. Software Development, 9(8), 28-35.

Gartner. (2015). Design thinking can revolutionize your customer experience strategies. White Paper, November 2015.

Gerber, T., Theorin, A., \& Johnsson, C. (2014). Towards a seamless integration between process modeling descriptions at business and production levels: Work in progress. Journal of Intelligent Manufacturing, 25(5), 1089-1099.

Gero, J. S., \& Kannengiesser, U. (2003). Function-behaviour-structure: A model for social situated agents. In Workshop on Cognitive Modeling of Agents and Multi-Agent Interactions, International Joint Conference on Artificial Intelligence, Acapulco, Mexico (pp. 101-107).

Gero, J. S., \& Kannengiesser, U. (2014). The function-behaviour-structure ontology of design. In A. Chakrabarti, \& L. T. M. Blessing (Eds.), An anthology of theories and models of design (pp. 263-283). Springer.

Grosskopf, A., Edelman, J., \& Weske, M. (2010). Tangible business process modelingmethodology and experiment design. In BPM 2009 Workshops (pp. 489-500). Berlin: Springer.

Harmon, P. (2016). The state of business process management 2016. BPTrends Report, Business Process Trends. 
Hoppe, S. (2014). Standardisierte horizontale und vertikale Kommunikation: Status und Ausblick. In T. Bauernhansl, et al. (Eds.), Industrie 4.0 in Produktion, Automatisierung und Logistik (pp. 325-341). Springer.

ISO 29481-3. (2010). Building information modelling-information delivery manual-Part 3: Model view definitions. Retrieved August 18, 2016, from http://www.freestd.us/soft4/2085144. $\mathrm{htm}$.

Kampert, D., \& Epple, U. (2014). Outside-in: Simplifying systems by integrating the outside perspective. In 2014 11th International Multi-Conference on Systems, Signals \& Devices (SSD), Barcelona (pp. 1-6).

Kannengiesser, U. (2014). Supporting value stream design using S-BPM. In S-BPM-ONEScientific Research (Vol. 170, pp. 151-160). LNBIP. Springer.

Kannengiesser, U. (2015). Integrating cross-organisational business processes based on a combined S-BPM/DSM approach. In 17th IEEE Conference on Business Informatics, Lisbon, Portugal.

Kannengiesser, U., Neubauer, M., \& Heininger, H. (2015). Subject-oriented BPM as the glue for integrating enterprise processes in smart factories. In On the Move to Meaningful Internet Systems: OTM 2015 Workshops (Vol. 9416, pp. 77-86). LNCS. Springer.

Kannengiesser, U., Neubauer, M., \& Heininger, H. (2016). Integrating business processes and manufacturing operations based on S-BPM and B2MML, S-BPM ONE 2016. Germany: Erlangen.

Kannengiesser, U., \& Roxin, A. (2016). An agile process modelling approach for BIM projects. In European Conference on Product and Process Modelling 2016, Cyprus.

Kocbek, M., Jošt, G., Heričko, M., \& Polančič, G. (2015). Business process model and notation: The current state of affairs. Computer Science and Information Systems, 12(2), 509-539.

Lass, S., \& Kotarski, D. (2014). IT-Sicherheit als besondere Herausforderung von Industrie 4.0. In W. Kersten, H. Koller, \& H. Lödding (Eds.), Industrie 4.0: Wie intelligente Vernetzung und kognitive Systeme unsere Arbeit verändern (pp. 397-419). Berlin: Gito.

Lawall, A., Reichelt, D., \& Schaller, T. (2015). Resource management and authorization for cloud services. In S-BPM ONE'15. New York: ACM Press. Article No. 18.

Lee, G., Park, Y. H., \& Ham, S. (2013). Extended process to product modeling (xPPM) for integrated and seamless IDM and MVD development. Advanced Engineering Informatics, 27, 636-651.

Mellor, S. J., Clark, A. N., \& Futagami, T. (2003). Model-driven development. IEEE Software, 20 (5), 14-18.

Moody, D. L. (2009). The "physics" of notations: Towards a scientific basis for constructing visual notations in software engineering. IEEE Transactions on Software Engineering, 35(5), 756778.

Müller, H. (2012). Using S-BPM for PLC code generation and extension of subject-oriented methodology to all layers of modern control systems. In C. Stary (Ed.), S-BPM One-Scientific Research (Vol. 104, pp. 182-204). LNBIP. Berlin: Springer.

Petre, M. (2009). Insights from expert software design practice. In 7th Joint Meeting of the European Software Engineering Conference (ESEC) and the ACM SIGSOFT Symposium on the Foundations of Software Engineering (FSE), Amsterdam, The Netherlands (pp. 233-242).

Recker, J. C. (2010). Opportunities and constraints: The current struggle with BPMN. Business Process Management Journal, 16(1), 181-201.

Rosemann, M. (2006). Potential pitfalls of process modeling: Part B. Business Process Management Journal, 12(3), 377-384.

Sadeghi, A.-R., Wachsmann, C., \& Waidner, M. (2015). Security and privacy challenges in industrial internet of things. In DAC'15, San Francisco, CA.

Saleh, J. H., Hastings, D. E., \& Newman, D. J. (2003). Flexibility in system design and implications for aerospace systems. Acta Astronautica, 53(12), 927-944.

Schilling, M. A. (2000). Toward a general modular systems theory and its application to interfirm product modularity. Academy of Management Review, 25(2), 312-334. 
Schilling, M. A., \& Steensma, H. K. (2001). The use of modular organizational forms: An industry-level analysis. Academy of Management Review, 44(6), 1149-1168.

Schön, D., \& Wiggins, G. (1992). Kinds of seeing and their functions in designing. Design Studies, 13(2), 135-156.

Schütze, M., Sachse, P., \& Römer, A. (2003). Support value of sketching in the design process. Research in Engineering Design, 14(2), 89-97.

Sinur, J., Odell, J., \& Fingar, P. (2013). Business process management: The next wave. Tampa: Meghan-Kiffer Press.

Turetken, O., \& Demirors, O. (2013). Business process modeling pluralized. In H. Fischer \& J. Schneeberger (Eds.), S-BPM ONE 2013, CCIS 360 (pp. 34-51). Berlin: Springer.

Ulrich, K. (1995). The role of product architecture in the manufacturing firm. Research Policy, 24 (3), 419-440.

van Aken, J. E. (2007). Design science and organization development interventions: Aligning business and humanistic values. Journal of Applied Behavioral Science, 43(1), 67-88.

Wooldridge, M., \& Jennings, N. R. (1995). Intelligent agents: Theory and practice. The Knowledge Engineering Review, 10(2), 115-152.

Wynn, D. C., Eckert, C. M., \& Clarkson, P. J. (2007). Modelling iteration in engineering design. In International Conference on Engineering Design (ICED'07), Paris, France, paper no. 561.

Yauch, C. A. (2007). Team-based work and work system balance in the context of agile manufacturing. Applied Ergonomics, 38(1), 19-27.

Open Access This chapter is distributed under the terms of the Creative Commons AttributionNonCommercial 4.0 International License (http://creativecommons.org/licenses/by-nc/4.0/), which permits any noncommercial use, duplication, adaptation, distribution and reproduction in any medium or format, as long as you give appropriate credit to the original author(s) and the source, provide a link to the Creative Commons license and indicate if changes were made.

The images or other third party material in this chapter are included in the work's Creative Commons license, unless indicated otherwise in the credit line; if such material is not included in the work's Creative Commons license and the respective action is not permitted by statutory regulation, users will need to obtain permission from the license holder to duplicate, adapt or reproduce the material. 